Rev. Adm. Saúde - Vol. 18, № 70, jan. - mar. 2018

http://dx.doi.org/10.23973/ras.70.73

RESENHA

\title{
O valor da inovação: criar o futuro do sistema de saúde (livro)
}

The value of innovation: creating the future of the health system (book)

\section{Matheus Guerra1}

1. Mestre em saúde pública. Estrategista de saúde pública no Departamento de Gestão do Conhecimento do Conselho do Distrito de Blekinge, Suécia

\section{RESUMO}

O livro "O valor da inovação: criar o futuro do sistema de saúde" revela o poder da inovação na transformação dos sistemas de saúde. Os resultados apresentados pelo autor mudam o foco das novas tecnologias para a inovação organizacional. Dentro deste novo contexto, o valor da inovação ultrapassa o desempenho do sistema de saúde para promover a coesão social, a competitividade e o desenvolvimento econômico.

Palavras-chave: sistema de saúde; hospitais; inovação; tecnologias

\section{ABSTRACT}

The book "The value of innovation: create the future of the health system" reveals the power of innovation in the transformation of the health systems. The results presented by the author shift the focus from the new technologies to organizational innovation. Within this new context, the value of innovation goes beyond the health system's performance to promote social cohesion, competitiveness and economic development.

Keywords: health system; hospitals; innovation; technologies 
O livro "O valor da inovação: criar o futuro do sistema de saúde" revela o poder da inovação na transformação do sistema de saúde. Os resultados apresentados ao longo do livro apontam para uma mudança do habitual foco das novas tecnologias para a inovação organizacional. As inovações com maior impacto na nossa sociedade, como por exemplo os serviços online ou através do telefone celular, envolvem necessariamente a reorganização estrutural dos diferentes sectores da economia. Enquanto muitas destas inovações têm uma importante componente tecnológica, os novos modelos organizacionais assumem-se como a chave para tirar o máximo partido do seu potencial.

A partir da análise do sistema de saúde, Casimiro Cavaco Dias reconhece que este manteve a sua estrutura praticamente inalterada nas últimas décadas. $O$ livro destaca as importantes oportunidades para a inovação organizacional e reestruturação do sistema de saúde ao longo de uma cadeia de valor. Tal significa uma mudança de sistema fragmentado e reativo a episódios isolados de doença aguda, para um modelo integrado de serviços de saúde orientados em torno da pessoa.

O autor debate as principais questões sobre inovação em saúde ao longo de doze capítulos que se organizam em quatro partes. Na primeira parte são apresentadas as principais questões de investigação, o modelo conceitual assim como os métodos de investigação utilizados no estudo. Na segunda parte, o autor analisa e discute os objetivos da inovação em saúde, assim como os seus principais motores. O quarto capítulo foca-se nos principais objetivos da inovação criada e implementada pelos hospitais. Os resultados revelam a melhoria de desempenho e a criação de novos conhecimentos e serviços como os dois principais objetivos. No quinto capítulo são analisados os principais motores da inovação em saúde incluindo as novas tecnologias, a cooperação externa, a estrutura e organização do trabalho, e desenvolvimento profissional. No sexto e sétimo capítulo analisam-se os resultados sobre as novas competências necessárias a inovação. Enquanto o sexto capítulo centrase nas competências organizacionais, o capítulo seguinte olha para as competências individuais e investimentos em desenvolvimento profissional.

$\mathrm{Na}$ última parte do livro são apresentadas as principais conclusões e implicações do estudo.

As principais medidas para transformar o sistema de saúde através da inovação são identificadas e priorizadas por um grupo de peritos. As doze medidas descritas são classificadas em termos de potencial impacto e dificuldade de implementação para apontar os cenários na criação do futuro da saúde em Portugal. Tornar o setor da saúde mais inovador e resiliente a futuras crises econômicas e financeiras implica o envolvimento dos diversos atores do sistema de saúde em torno do valor da inovação para a pessoa, o cidadão e o utilizador dos serviços de saúde. Assim o autor destaca uma série de implicações dirigida especificamente aos profissionais de saúde, decisores políticos, indústria tecnológica, universidades e centros de investigação, assim como para o utilizador de serviços e a comunidade. 
Este livro apresenta quatro importantes mensagens para a transformação do sistema de saúde através da inovação. Primeiro, destaca a capacidade de transformação do sistema de saúde como aspecto chave para assegurar a sua sustentabilidade. Os avanços no desenvolvimento do sistema de inovação em saúde têm potencial para desenvolver novos produtos e serviços mais efetivos na resposta aos novos desafios em saúde e às novas necessidades e expectativas dos cidadãos. Segundo, a transformação do sistema de saúde requer a criação de novos conhecimentos, assim como a sua utilização efetiva no cotidiano do sistema de saúde. Os hospitais mais inovadores apontam as novas tendências de transformação, revelando alguns sinais de como será o futuro do sistema de saúde. Terceiro, os resultados apresentados ao longo deste trabalho revelam a nova natureza da inovação em saúde: a inovação em saúde é cada vez mais aberta e centrada na pessoa. Deste modo, a cooperação externa assume-se como o motor central da inovação. Além do envolvimento de outros serviços de saúde e outros setores da sociedade, o sistema de saúde centra-se na pessoa enquanto cocriadora de inovação.

Por último, a inovação em saúde tem o potencial de aproximar a saúde da economia. De fato, ainda que o setor da saúde seja muitas vezes considerado apenas como consumidor de recursos, o desenvolvimento de inovação em saúde tem impacto significativo na melhoria do desempenho do sistema de saúde, maior coesão social, maior competitividade e maior desenvolvimento econômico. Face à crise económica e financeira atual, o setor da saúde deve assumir o seu papel central no relançamento da economia.

\section{REFERÊNCIAS}

Cavaco Dias, Casimiro. O valor da inovação: criar o futuro do sistema de saúde. Coimbra: Edições Almedina; 2015. 213p.

Recebido: 20 de dezembro de 2017. Publicado: 12 de janeiro de 2018

Correspondência: Matheus Guerra, Department of Knowledge Support, Blekinge County Council, Wämö Center, 37181 Karlskrona, Sweden. E-mail: enspusp@gmail.com

Conflito de Interesses: o autor declara não haver conflito de interesses. 
(C) This is an Open Access article distributed under the terms of the Creative Commons Attribution License, which permits unrestricted use, distribution, and reproduction in any medium, provided the original work is properly cited 
